\title{
Double-shock control bump design optimization using hybridized evolutionary algorithms
}

\author{
D S Lee ${ }^{1 *}$, L F Gonzalez ${ }^{2}$, J Periaux ${ }^{1,3}$, and G Bugeda ${ }^{1,3}$ \\ ${ }^{1}$ International Center for Numerical Methods in Engineering (CIMNE), Barcelona, Spain \\ ${ }^{2}$ Australian Research Centre Aerospace Automation (ARCAA) School of Engineering System, Queensland University of \\ Technology, Australia \\ ${ }^{3}$ Universidad Politécnica de Cataluña, Barcelona, Spain
}

The manuscript was received on 29 October 2010 and was accepted after revision for publication on 16 March 2011.

DOI: $10.1177 / 0954410011406210$

\begin{abstract}
This study investigates the application of two advanced optimization methods for solving active flow control (AFC) device shape design problem and compares their optimization efficiency in terms of computational cost and design quality. The first optimization method uses hierarchical asynchronous parallel multi-objective evolutionary algorithm and the second uses hybridized evolutionary algorithm with Nash-Game strategies (Hybrid-Game). Both optimization methods are based on a canonical evolution strategy and incorporate the concepts of parallel computing and asynchronous evaluation. One type of AFC device named shock control bump (SCB) is considered and applied to a natural laminar flow (NLF) aerofoil. The concept of SCB is used to decelerate supersonic flow on suction/pressure side of transonic aerofoil that leads to a delay of shock occurrence. Such active flow technique reduces total drag at transonic speeds which is of special interest to commercial aircraft.

Numerical results show that the Hybrid-Game helps an EA to accelerate optimization process. From the practical point of view, applying a SCB on the suction and pressure sides significantly reduces transonic total drag and improves lift-to-drag $(L / D)$ value when compared to the baseline design.
\end{abstract}

Keywords: active flow control, shock control bump, shape design optimization, Hybrid-Game, Nash equilibrium, evolutionary algorithm

\section{INTRODUCTION}

Developing an efficient optimization technique is still one of the most challenging tasks in the field of evolutionary algorithm (EA) research. As modern engineering problems become progressively more complex not only robust but also efficient tools are required. One of the emerging techniques to improve an optimization performance can be the

\footnotetext{
*Corresponding author: International Center for Numerical Methods in Engineering (CIMNE), Edificio C1, Gran Capitan, 08860 Barcelona, Spain.

email:ds.chris.lee@gmail.com
}

use of Nash-equilibrium concept which will be acting as a pre-conditioner of global optimizer.

Lee et al. [1] studied the concept of Hybrid-Game (Pareto + Nash) coupled to a well-known multiobjective evolutionary algorithm (MOEA); non-dominating sort genetic algorithm II (NSGA-II) [2] to solve unmanned aerial system multi-objective mission path planning system design problems. Their research shows that the Hybrid-Game improves the NSGA-II performance by 80 per cent when compared to the original NSGA-II. In addition, Lee et al. [3] hybridized NSGA-II with Nash-Game strategy to study a role of Nash-Players in Hybrid-Game by solving multi-objective mathematical test cases; 
non-uniformly distributed non-convex, discontinuous, and mechanical design problem. Their research also shows that hierarchical asynchronous parallel multi-objective evolutionary algorithm (HAPMOEA) [4] can also be hybridized to solve a real-world robust multidisciplinary design problem. Numerical results show that the Hybrid-Game improves 70 per cent of HAPMOEA performance while producing better Pareto optimal solutions. References [1, 3, 5] clearly describe the merits of using Hybrid-Game coupled to MOEA for engineering design applications which consider a complex geometry or a large number of design variables.

Hybrid-Game has two major characteristics; the first is a decomposition of design problem, a multi-objective design problem for instance can be split into several simpler single-objective problems which correspond to Nash-Players which have their own design search space. The second temperament is that Nash-Players are synchronized with a Global/ Pareto-Player as a pre-conditioner; hence, ParetoPlayer can accelerate the optimization process using a set of elite designs obtained by the Nash-Players


optimization. nain goal of this study is of Hybrid-Game

Nash-Player. In this study, HAMOPEA is hybridized with Nash game strategy to improve optimization Registernfor free aptimetrastipwwwethedpardiandom mented to active flow control (AFC) device-shape design optimization and their performance are compared in terms of computational cost and design quality.

Recent advances in design tools, materials, electronics, and actuators offer implementation of flow control technologies to improve aerodynamic efficiency $[6-10]$. Such aerodynamic improvement saves mission operating cost while condensing critical aircraft emissions. The main benefits of using ACF techniques on current transonic aircraft are to improve aerodynamic efficiency and reduce manufacturing cost when compared to designing a new airfoil or wing planform shape.

In this study, one of AFC devices; double-shock control bump (SCB) [8-10] is applied on the suction and pressure sides of a natural laminar flow (NLF) aerofoil; the RAE $5243[\mathbf{1 0}, \mathbf{1 1}]$ to reduce transonic total drag, especially wave drag at the critical flight conditions where two shocks occur.

The rest of the article is organized as follows; section 2 describes the optimization methods: HAPMOEA and Hybrid-Game. Section 3 presents mathematical benchmarks using Hybrid-Game.
Section 4 demonstrates the use of a SCB. Section 5 considers double-SCB design optimization using HAPMOEA and Hybrid-Game. Section 6 delivers conclusion and future works.

\section{OPTIMIZATION METHODS}

The EA used in this study is based on covariance matrix adaptation evolutionary strategies (CMA-ES) $[12,13]$ which incorporates an asynchronous parallel computation and a Pareto tournament selection [1416]. The first method; HAPMOEA uses the concept of hierarchical multi-population topology which can handle different models including precise, intermediate, and approximate models. Each node (Node0Node6) belonging to the different hierarchical layer can be handled by a different EA code, as shown in Fig. 1(a).

The second method hybridizes HAPMOEA by applying a concept of Nash-Equilibrium instead of the concept of hierarchical multi-population topology $[4,17]$ which is denoted as Hybrid-Game. Figure 1(b) shows one example topology for Hybrid-
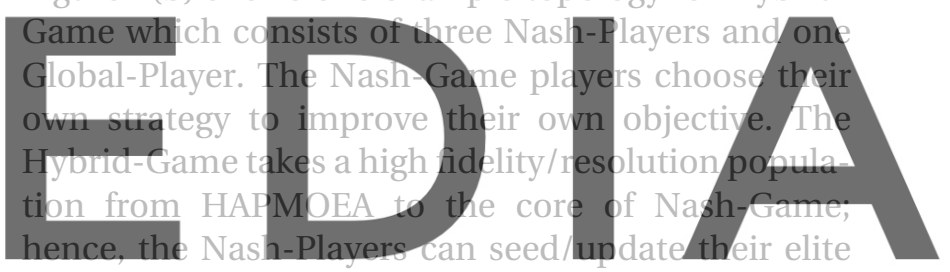

designs to Global-Player (Node0)

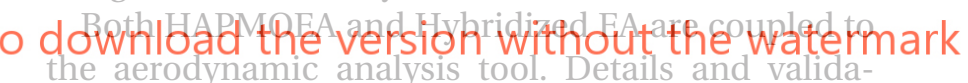

tions of HAPMOFA and Hybrid

in references $[\mathbf{1}, \mathbf{3}, \mathbf{1 7}]$. Lee et al. $[\mathbf{3}, \mathbf{1 7}]$ described the details of topology for HAPMOEA and Hybrid-Game for robust multidisciplinary design problem, and showed their validation by solving multi-objective mathematical design problems including nonuniformly distributed non-convex, discontinuous (TNK), and mechanical design problems.

\section{MATHEMATICAL BENCHMARKS}

In this section, Hybrid-Game is implemented to NSGA-II [2] to solve two complex mathematical design problems; single-objective mathematical design developed by author and Zitzler, Deb, and Thiele (ZDT6) [18] are considered. Both NSGA-II and Hybrid-Game use same optimization parameters: a constant random seed, population size $=100$, cross-over rate $=0.9$, and mutation probability $=1 / n$ where $n$ is the number of decision variables. The reason why a constant random seed is considered is to produce the same initial random population for both NSGA-II and Hybrid-Game. 
(a)
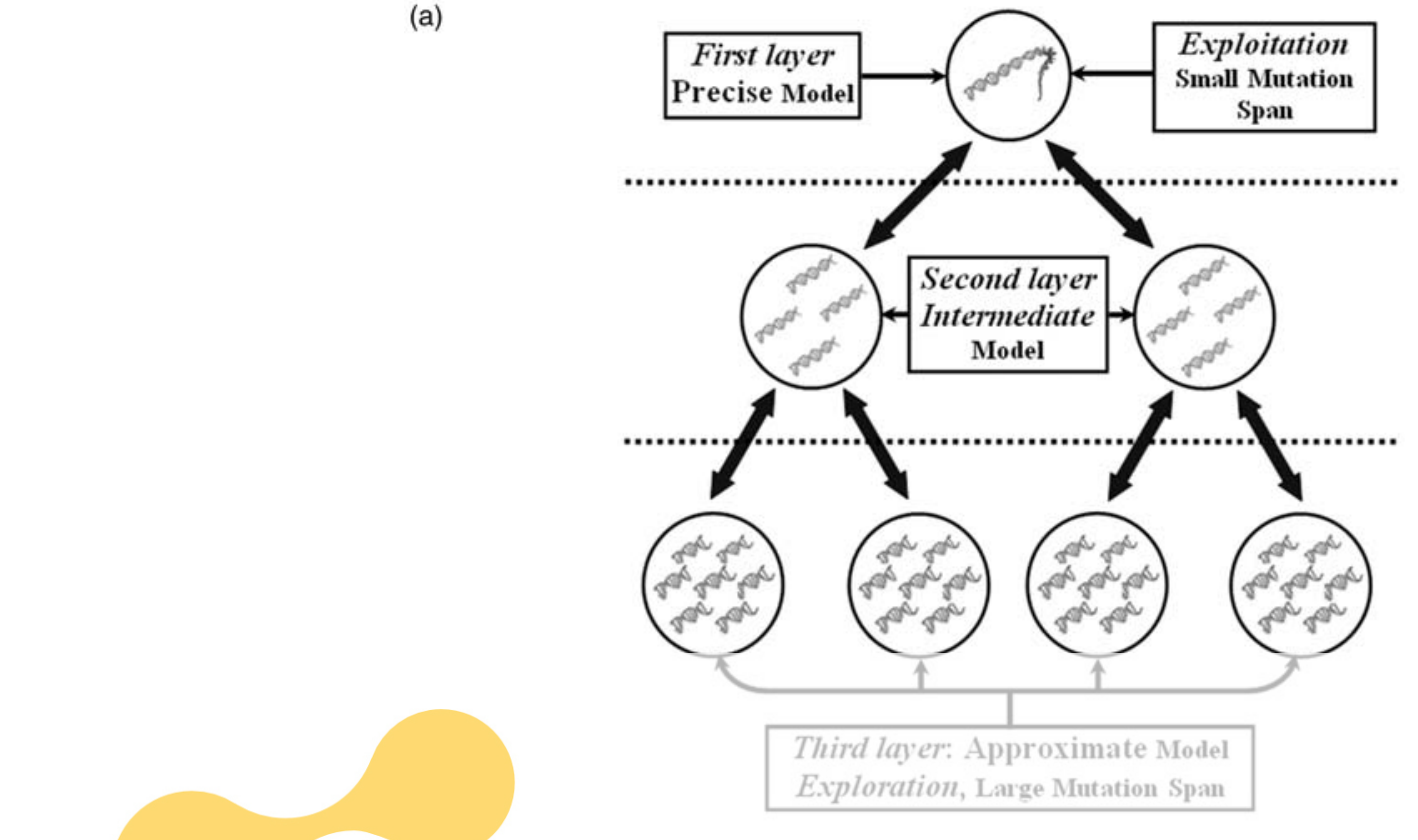

(b)
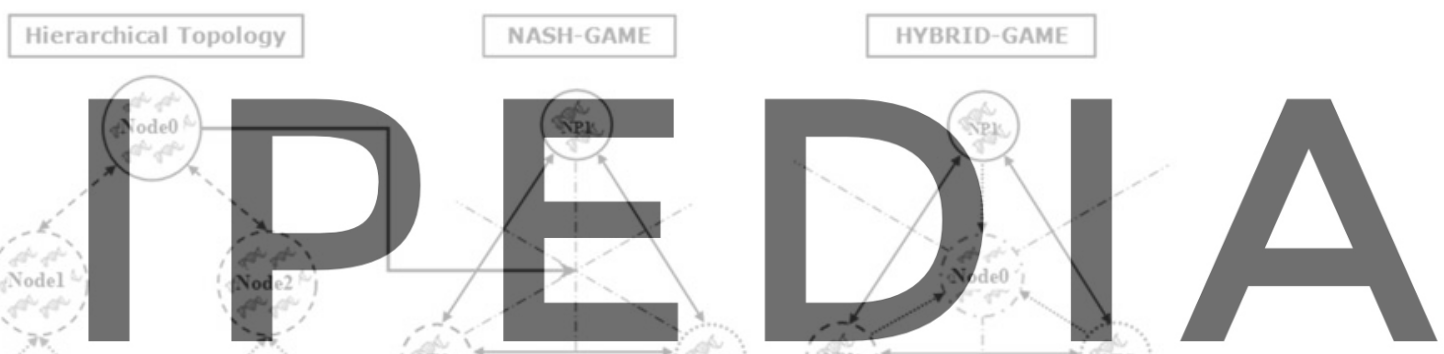

Register for free at https//www.scipedia,com to download the version without the watermark

Fig. 1 (a) Hierarchical multi-population topology and (b) example topology of Hybrid-Game

\subsection{Single-objective mathematical design optimization using NSGA-II and Hybrid-Game}

One single-objective mathematical design problem which is similar to inverse design (desired to have zero value for fitness function) is considered. The fitness function is shown (1). Two test cases are conducted with different number of design variables ( $n=20, n=30$ ). The same random initial population is used for both NSGA-II and Hybrid-Game. HybridGame employs three players: one Global-Agent (GlobalP) minimizing equation (1) and two NashAgents (NashP1 and NashP2) minimizing equations (2) and (3). The stopping criterion for NSGA-II and Hybrid-Game is when the fitness value reaches lower than predefined value $1.0 \times 10^{-6}$, i.e. $f_{\text {MOGA }}$ and $f_{\text {HMOGA }} \leqslant 1.0 \times 10^{-6}$.

$$
\begin{aligned}
& f_{\text {Global-Player }}\left(x_{i}\right)=\sum_{i=2}^{n}\left(x_{i}-0.5\right)^{2} \\
& f_{\text {Nash-Player1 }}\left(x_{i}, x_{i}^{*}\right)= \sum_{i=1}^{n_{\text {NashP1 }}}\left(x_{i}-0.5\right)^{2} \\
&+\sum_{i=1}^{n_{\text {NashP2 }}}\left(x_{i}^{*}-0.5\right)^{2} \\
& f_{\text {Nash-Player2 }}\left(x_{i}^{*}, x_{i}\right)= \sum_{i=1}^{n_{\text {NashP1 }}}\left(x_{i}^{*}-0.5\right)^{2} \\
&+\sum_{i=1}^{n_{\text {NashP2 }}}\left(x_{i}-0.5\right)^{2}
\end{aligned}
$$




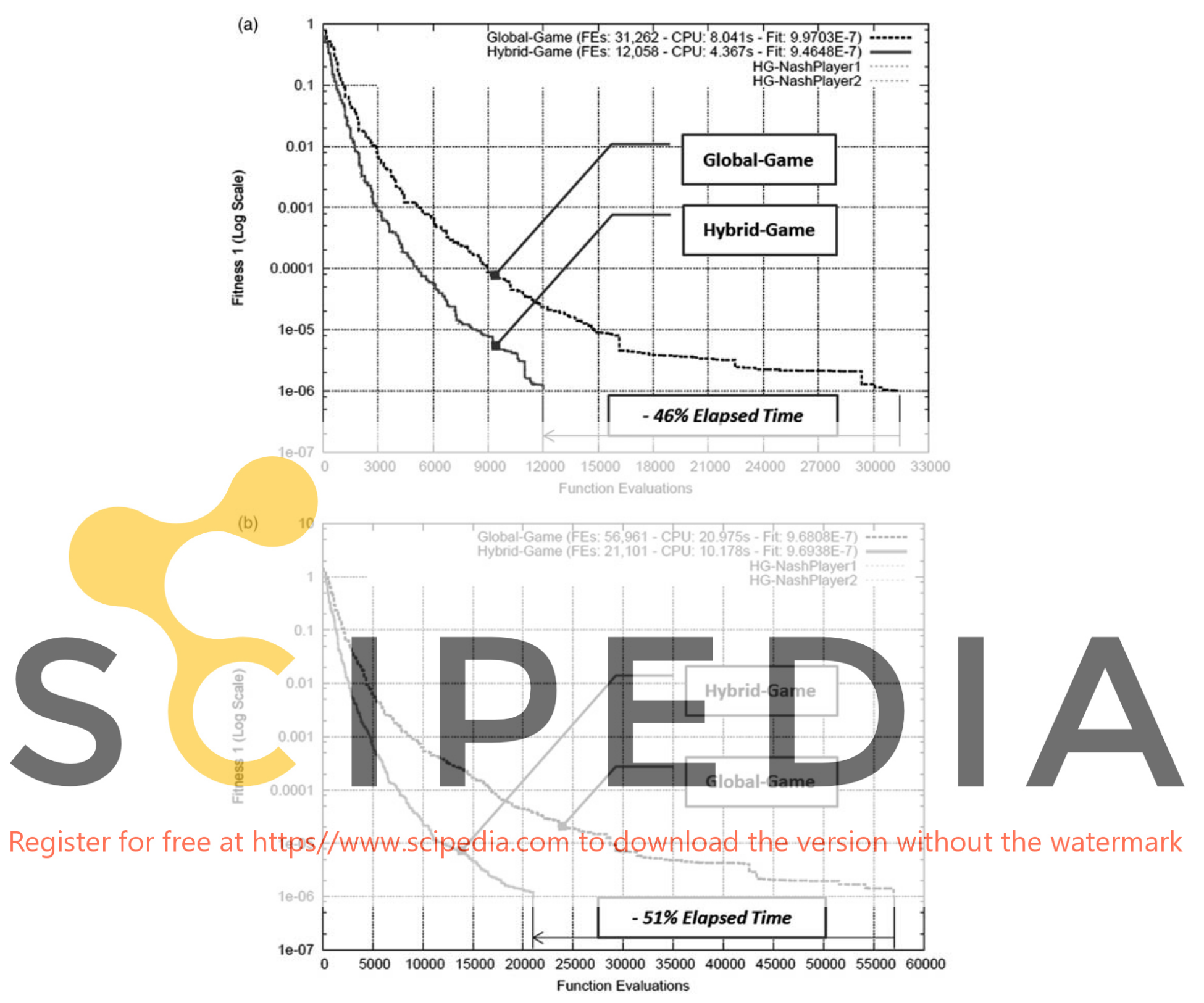

Fig. 2 Convergence history obtained by NSGA-II and Hybrid-Game for Testl ( $n_{\text {Global }}=20$ : top) and Test2 $\left(n_{\text {Global }}=30:\right.$ bottom $)$

where $n_{\text {Global }}=[20,30], n_{\mathrm{NashP} 1}=[10,15]$, and $n_{\text {NashP2 }}=[10,15] . x_{i}^{*}$ is an elite design obtained by the Nash-Player 1 and Nash-Player 2.

Figure 2 compares the convergence history obtained by NSGA-II and Hybrid-Game for ( $n=20$, $n=30$ ). It can be seen that Hybrid-Game has converged $\left(f \leqslant 1.0 \times 10^{-6}\right)$ faster than NSGA-II; for 20 design variables, Hybrid-Game converged after 12058 function evaluations (4.3 s) while NSGA-II converged after 31262 function evaluations (8.0 s). For the second test with 30 design variables, Hybrid-Game converged after 21101 function evaluations $(10.178 \mathrm{~s})$, while NSGA-II converged after 56961 function evaluations (20.975 s). It can be seen that Hybrid-Game can save almost 50 per cent of computational cost while converging at one-third of total function evaluations of NSGA-II.

\subsection{Multi-objective mathematical design problem using NSGA-II and Hybrid-Game}

For the multi-objective mathematical design, ZDT6 is considered [18]. It is formulated, as shown in equations (4) and (5).

$$
\begin{aligned}
& f_{1}\left(x_{1}\right)=1-\exp \left(-4 x_{1}\right) \sin ^{6}\left(6 \pi x_{1}\right) \\
& f_{2}\left(f_{1}, g\right)=1-\left(f_{1} / g\right)^{2}
\end{aligned}
$$




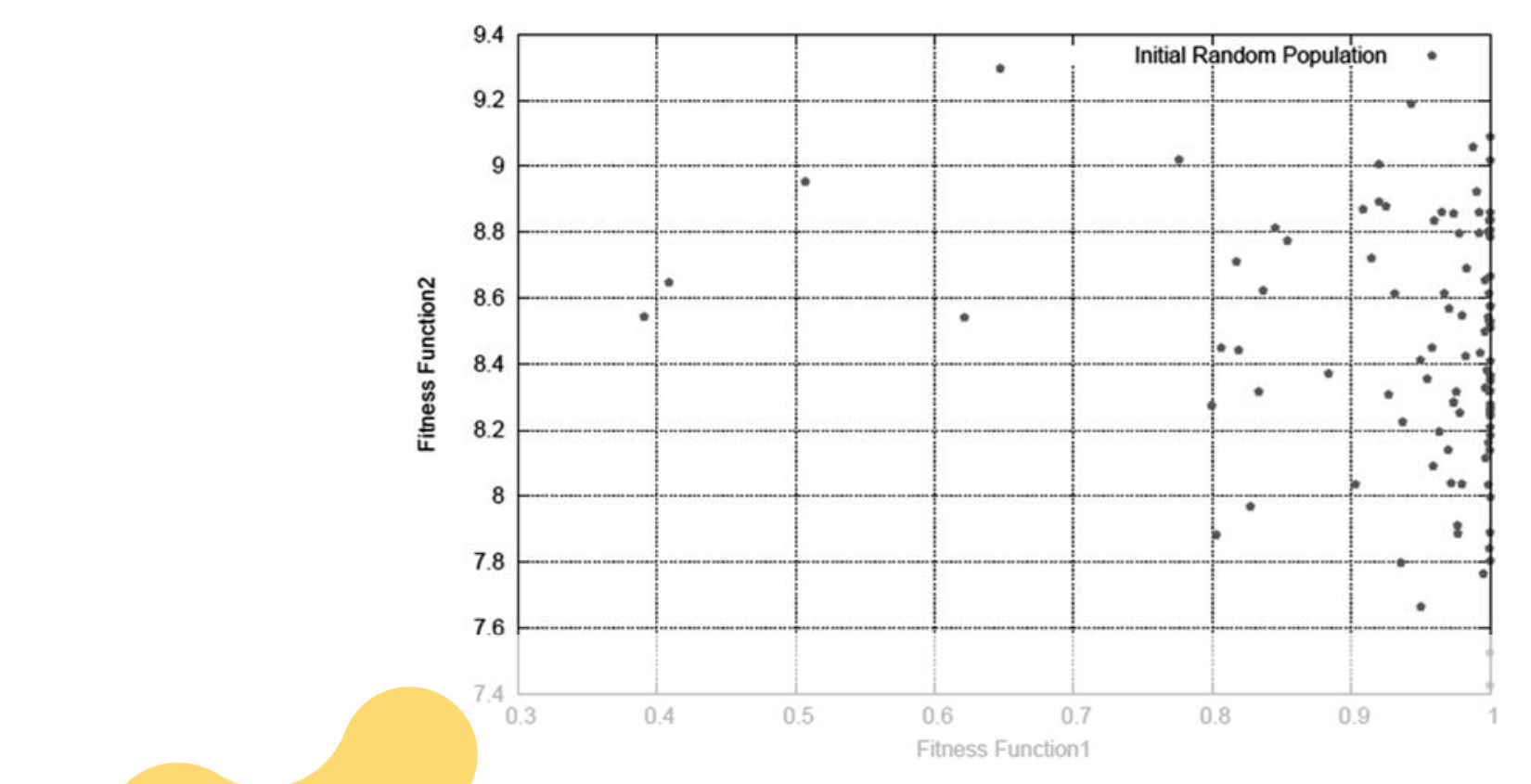

Fig. 3 Initial random population for ZDT6 obtained by NSGA-II and Hybrid-Game

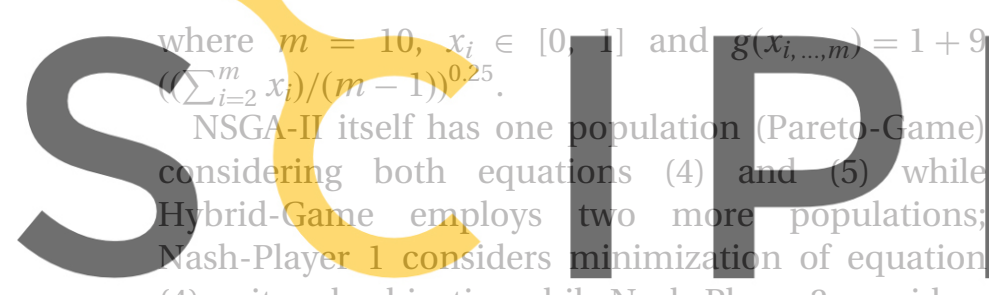

(4) as its sole objective while Nash-Player 2 considers Register mimization of equation (5) with. fixed.elite design Register for free at https www.scipedia.com to

$$
f_{N P 2}\left(f_{N P 1}, g\right)=1-\left(f_{N P 1}\left(x_{1}^{*}\right) / g\right)^{2}
$$

In this problem, Nash-Game splits the ZDT6 into two simpler problems corresponding to NashPlayer 1 and Nash-Player 2. In addition, the elite designs; here, $x_{1}^{*}, \ldots, x_{10}^{*}$ obtained by Nash-Players 1 and 2 will be seeded to the Pareto-Player population (original population of NSGA-II). Due to the constant random seed, NSGA-II and Hybrid-Game produce the same initial random population, as shown in Fig. 3. In addition, the optimization using NSGA-II is stopped after 200 generations, while Hybrid-Game is stopped when HybridGame reached the computational cost of NSGA-II. These conditions will provide to make a fair comparison.

Pareto optimal fronts obtained by NSGA-II (after 100 and 200 generations) and Hybrid-Game (after 5 and $13 \mathrm{~s}$ ) are compared, as shown in Fig. 4(a) and (b). It can be seen that both NSGA-II and Hybrid-Game are converging to the same solutions; however, the Pareto-Game of Hybrid-Game has much better solutions for both objectives after 100 and 200 generations

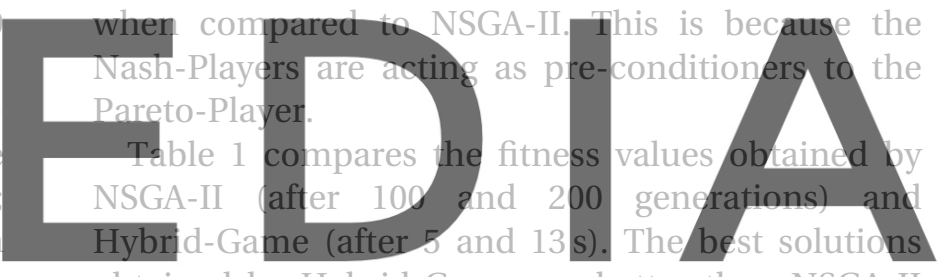

obtained by Hybrid-Game are better than NSGA-II duse to injection of the elite design obtained by Hybrid-Game. In other words, the use of HybridGame (Nash + Pareto) improves the optimization efficiency of NSGA-II due to the two major characteristics; the decomposition of design problem and the pre-conditioning.

\section{WAVE DRAG REDUCTION VIA SCB}

At transonic speed, the flow over aircraft wing causes shock waves where there is a large amount of gas property changes and the flow becomes irreversible. Through the shock, total pressure decreases and entropy increases which means there is a loss of energy. In other words, there is an increment of wave drag. To cope with this problem, Ashill et al. [8] proposed the concept of a transonic bump which is so-called SCB using geometry adoption on an aerofoil. As illustrated in Fig. 5, the typical design variables for SCB are: length, height, and peak position and, the centre of SCB will be located at sonic point where the flow speed transits from supersonic to subsonic on the transonic aerofoil design. 

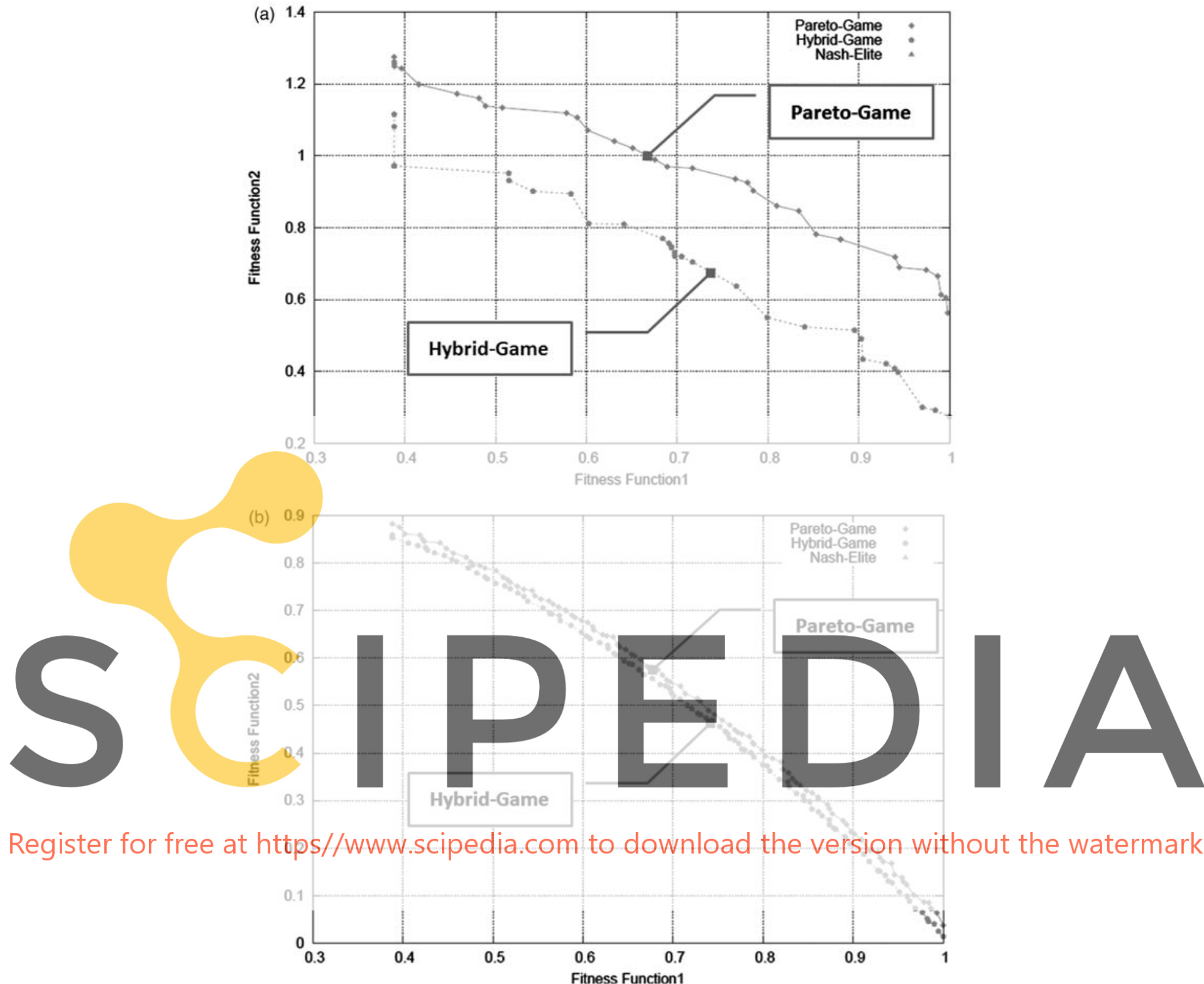

Fig. 4 (a) Pareto optimal front obtained by NSGA-II: (a) 100 generations, elapsed time: $5 \mathrm{~s}$ and Hybrid-Game (89 generations) and (b) 200 generations, elapsed time: 13 s and Hybrid-Game (160 generations)

Table 1 Comparison of fitness values obtained by NSGA-II and HNSGA-II for ZDT6

\begin{tabular}{|c|c|c|c|c|}
\hline \multirow[b]{3}{*}{ Game strategies } & \multirow{3}{*}{$\begin{array}{l}\begin{array}{l}\text { Non-dominating sort } \\
\text { genetic algorithm II }\end{array} \\
\text { Pareto-Game }\end{array}$} & \multicolumn{3}{|c|}{ Hybrid-Game (hybrid non-dominating sort genetic algorithm II) } \\
\hline & & \multirow[b]{2}{*}{ Pareto-Game } & \multicolumn{2}{|c|}{ Nash-Game } \\
\hline & & & Nash-Player1 & Nash-Player2 \\
\hline Best Fit1 (Gen100) & $0.38832,1.27476$ & $0.38832,1.11518$ & 0.38832 & 0.97123 \\
\hline Best Fit2 (Gen100) & $0.99790,0.56306$ & $0.99957,0.27448$ & & \\
\hline Best Fit1 (Gen200) & $0.38832,0.88232$ & $0.38832,0.85884$ & 0.38832 & 0.85296 \\
\hline Best Fit2 (Gen200) & $0.99999,0.03730$ & $0.99999,0.01392$ & & \\
\hline
\end{tabular}

Best Fit1 and Fit2 represent the best solutions for fitness functions 1 and 2, respectively. 


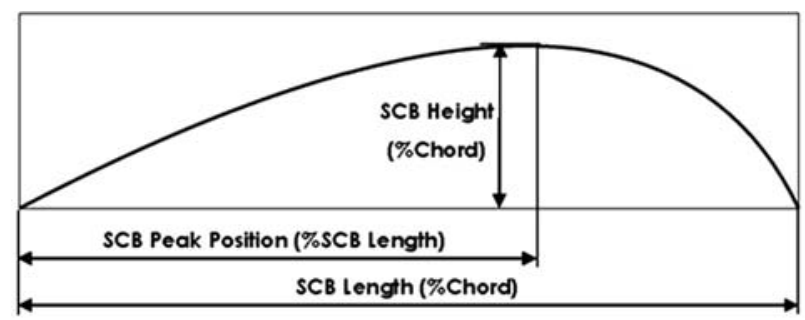

Fig. 5 Design components of SCB

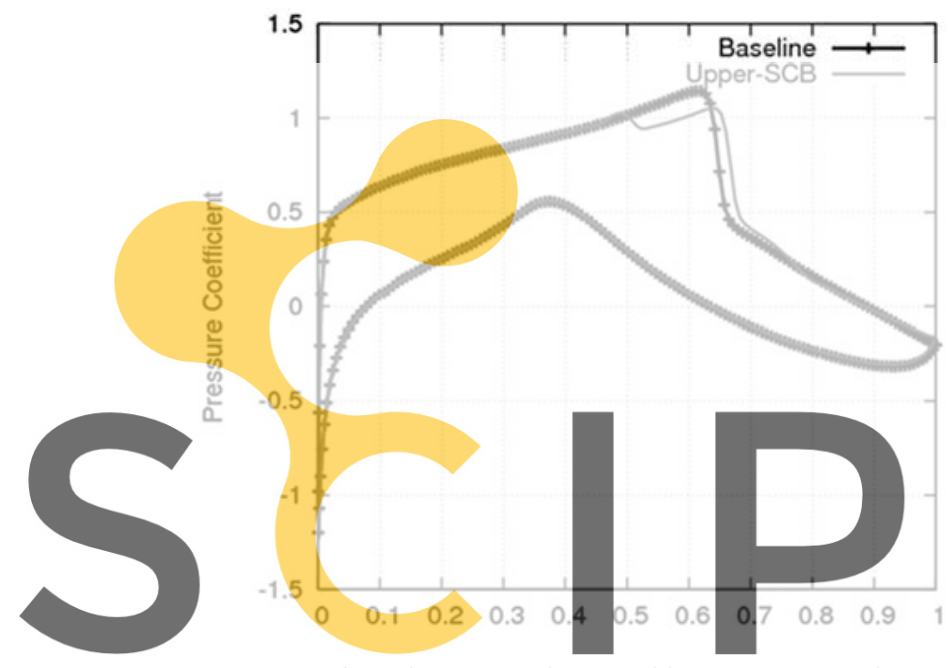

Fig.6 Cp distributions obtained by RAE 2822 (dots and

Register for free at https//WwW.scipedia.com to

Figure 6 illustrates the $C p$ distributions obtained by RAE 2822 aerofoil and RAE 2822 with SCB. For aerodynamic analysis tool, MSES (Euler and boundary layer) written by Drela [19] is utilized. The transonic flow over normal aerofoil without SCB accelerates the supersonic and the pressure forms a strong shock that leads to a high-wave drag $\left(C d_{\text {Wave }}\right)$; however, the pressure difference over the SCB causes a deceleration of supersonic flow which delays shock occurrence. SCB cannot totally remove a shock; however, it makes a weaker shock or breaks into isentropic compression waves (lower $C d_{\text {wave }}$ ).

Table 2 compares the aerodynamic performance obtained by RAE 2822 and with SCB. Even though applying SCB on RAE 2822 produces 5 per cent higher viscous drag $\left(\Delta C d_{\text {Viscous }}=0.0005\right)$, it reduces 60 per cent wave $\operatorname{drag}\left(\Delta C d_{\text {Wave }}=0.0036\right)$ while improving 19 per cent of $L / D$ when compared to RAE 2822 aerofoil.

Applying SCB on either suction or pressure side of aerofoil will produce slightly thicker thickness ratio
Table 2 Aerodynamic characteristics

\begin{tabular}{lllll}
\hline Aerofoil & $C d_{\text {Total }}$ & $C d_{\text {Viscous }}$ & $C d_{\text {Wave }}$ & $L / D$ \\
\hline RAE 2822 & 0.0153 & 0.0093 & 0.0060 & 34.34 \\
With SCB & 0.0123 & 0.0098 & 0.0024 & 42.6 \\
& $(-20 \%)$ & $(+5 \%)$ & $(-60 \%)$ & $(+24 \%)$ \\
\hline
\end{tabular}

$M_{\infty}=0.77, R e=17.93 \times 10^{6}$ and $C_{l}$ is fixed to 0.524 .

$(t / c)$ which causes increment of viscous drag ( $\left.C d_{\text {Viscous }}\right)$; however, the use of SCB is still beneficial due to $C d_{\text {Wave }}$ reduction especially when the Mach number is higher than critical Mach number where the shock starts appearing.

In Section 5, the shape of SCB is optimized at critical flight conditions where two shocks occur on the suction and pressure sides of aerofoil. This flight conditions make a suitable application for Hybrid-Game (Global + Nash) since two SCBs are required. The aerodynamic characteristics of baseline with the optimal double-SCB are also investigated at normal flight conditions where a single shock is on the suction side
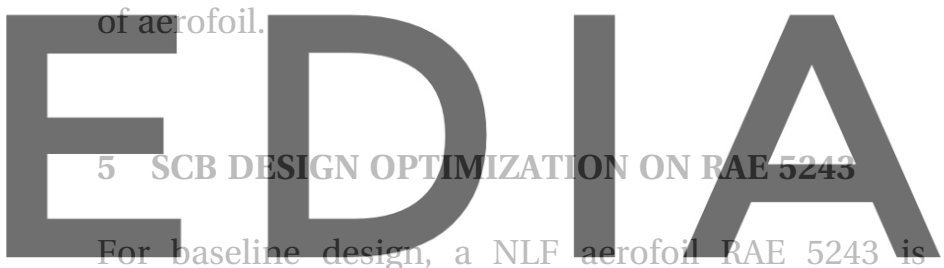

selected, as shown in Fig. 7(a). The problem considers

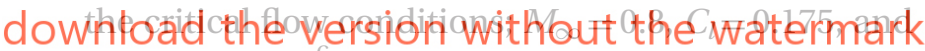
$R e=18.63 \times 10^{6}$ where two shocks occur on the suction and pressure sides of RAE 5243 aerofoil, as shown in Fig. 7(b).

The sonic points on the suction and pressure sides are occurred at 62.6 per cent and 58.1 per cent of chord, respectively. In the following sections, double-SCB design optimization using HAPMOEA and Hybrid-Game are conducted to minimize the total drag $\left(C d_{\text {Total }}\right)$. The aerodynamic analysis tool, MSES will run two times at each function evaluation; the first run will analyse SCB on the suction-side aerofoil and then two SCBs on both the suction and pressure sides will be analysed at the second run.

\subsection{Evaluation mechanism for HAPMOEA and Hybrid-Game}

Figure 8(a) shows the evaluation mechanism for HAPMOEA which consists of hierarchical multipopulation (Node0-Node6) based on multi-resolution. Each population will run aerodynamic analysis tool two times to evaluation double-SCB design in different resolution conditions. 
(a)

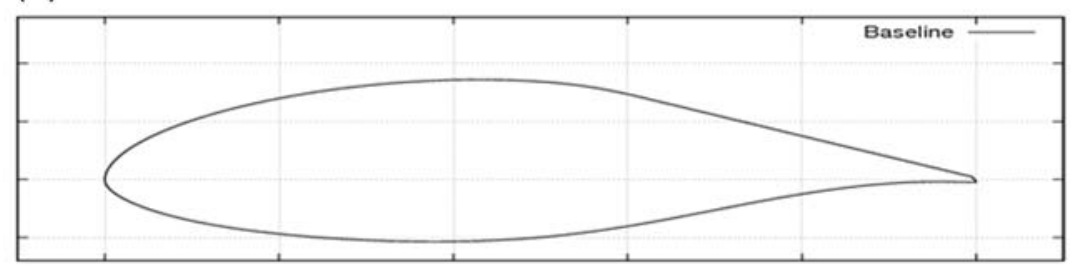

(b)

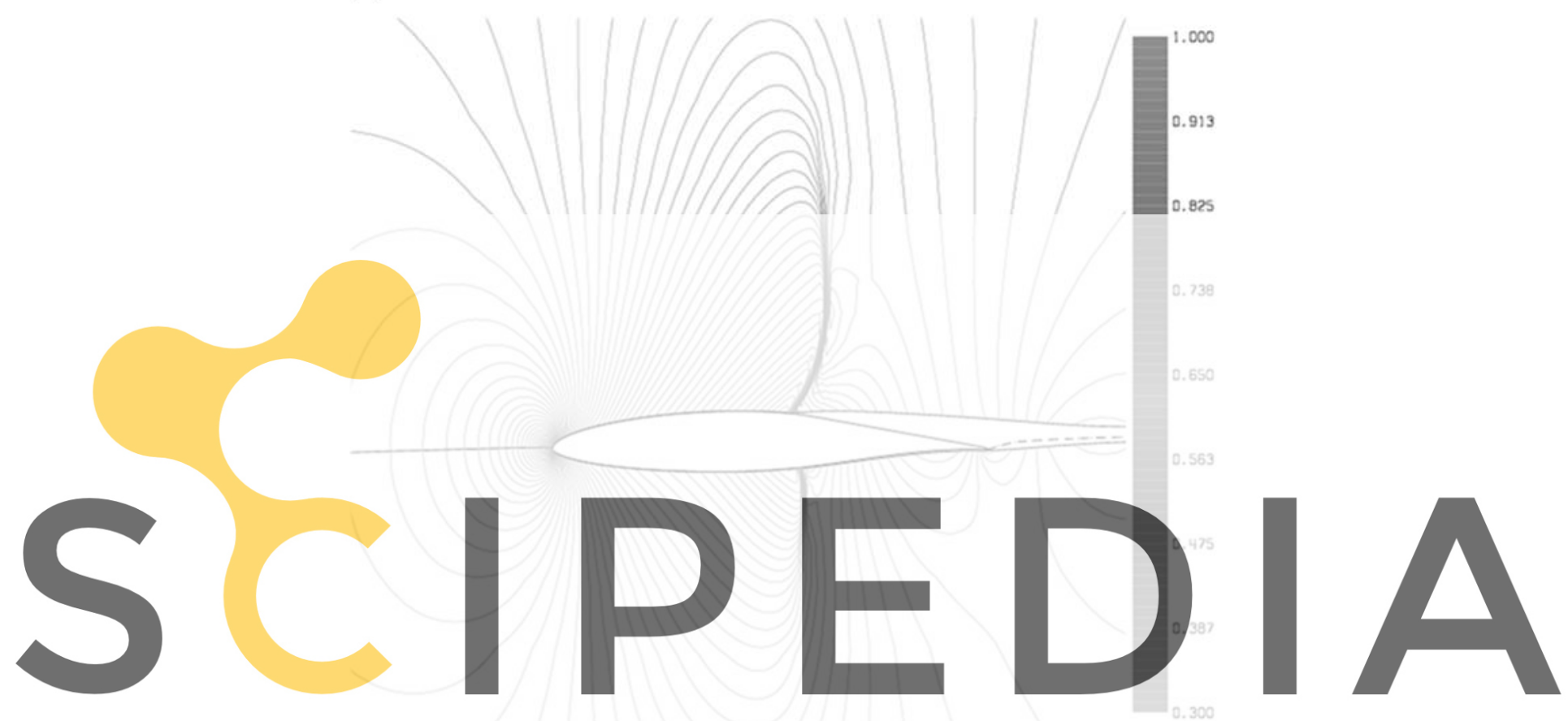

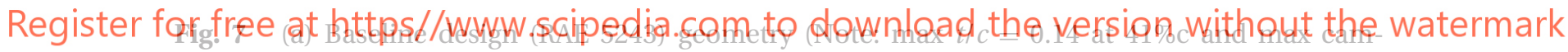
ber $=0.018$ at $54 \%$ c) and (b) $P / P_{0}$ contour of RAE 5243

Figure $8(\mathrm{~b})$ shows the evaluation mechanism for Hybrid-Game which employs three players: GlobalPlayer and Nash-Players 1 and 2. Solely, GlobalPlayer runs aerodynamic analysis tool two times since its optimization domain includes SCBs on both the suction and pressure sides. However, the analysis tool will run only once for Nash-Players 1 and 2 due to the Nash-Game characteristics, decomposition of design problem. For Hybrid-Game, double-SCB design problem becomes two singleSCB design problems; Nash-Game 1 will only optimize SCB on the suction side of aerofoil with elite SCB obtained by Nash-Player 2 on the pressure side, while Nash-Player 2 will optimize SCB on the pressure side of aerofoil with elite SCB design from NashPlayer 1 on the suction side. The elite designs obtained by Nash-Players will be seed to the population of the Global-Player that will allow Global-Player to accelerate optimization process.

\subsection{SCB design optimization using HAPMOEA}

\subsubsection{Problem definition}

This test case considers a single-objective doubleSCB design optimization using HAPMOEA to minimize total drag $\left(C d_{\text {Total }}\right)$ which consists of viscous drag $\left(C d_{\text {Viscous }}\right)$ and wave drag $\left(C d_{\text {Total }}\right)$. The flow conditions $M_{\infty}=0.8, C_{l}=0.175$, and $R e=18.63 \times 10^{6}$. The fitness function is shown in equation (7)

$$
f\left(\mathrm{SCB}_{\text {Suction }}, \mathrm{SCB}_{\text {Pressure }}\right)=\min \left(\mathrm{Cd}_{\text {Total }}\right)
$$

where $C d_{\text {Total }}=C d_{\text {Viscous }}+C d_{\text {Wave }}$.

\subsubsection{Design variables}

The design variables bound for both SCBs on the suction and pressure sides are illustrated in Table 3. 
(a)
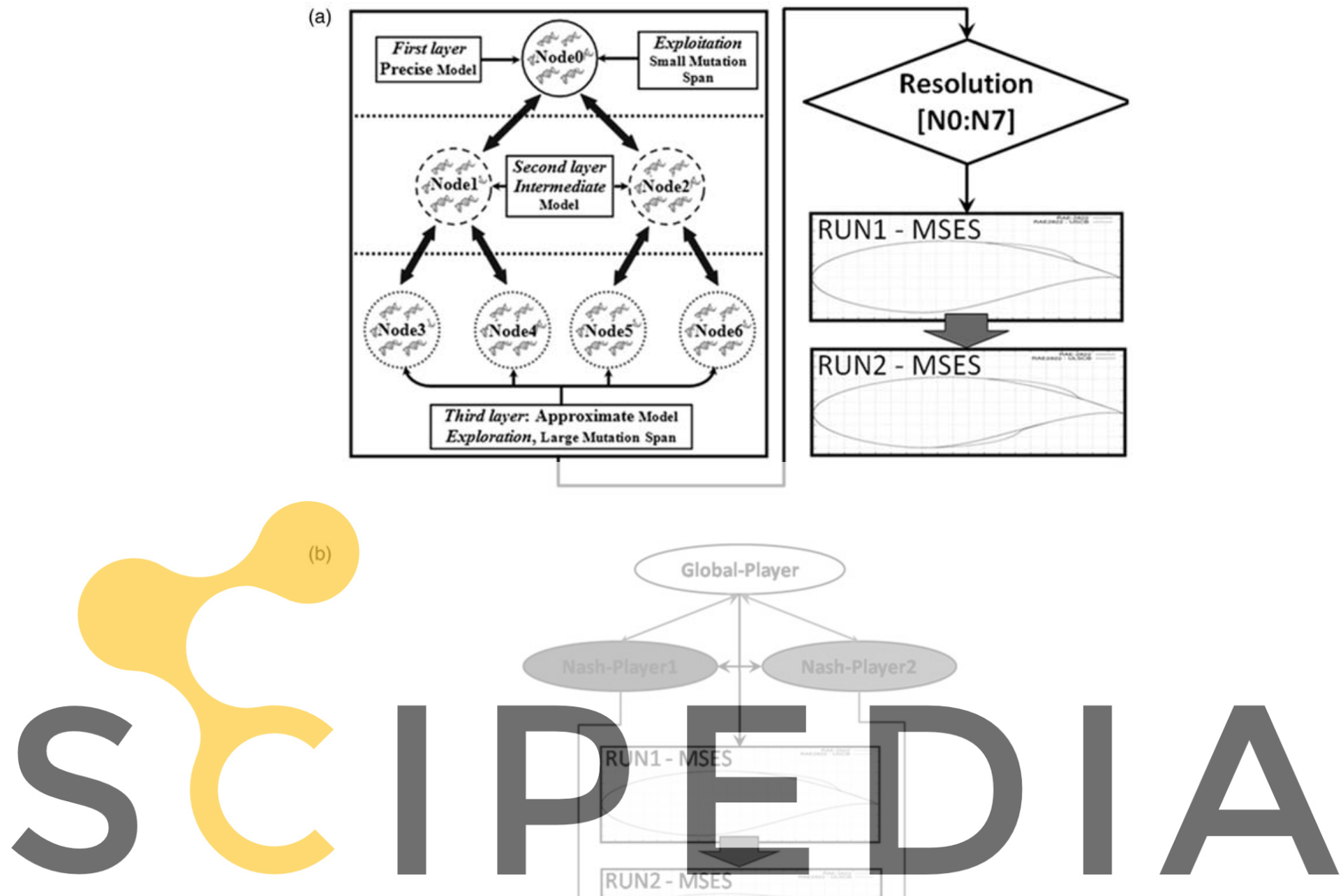

Register for free at https//www.scipedia.com to download the version without the watermark

Fig. 8 (a) Evaluation mechanism of: (a) HAPMOEA and (b) Hybrid-Game

Table 3 SCB design variables and bounds

\begin{tabular}{llc}
\hline Design variables & Lower bound & Upper bound \\
\hline Length (\% chord) & 15 & 30 \\
Height (\% chord) & 0.15 & 0.65 \\
Peak position & 0 & 100 \\
\hline
\end{tabular}

Peak position is in terms of percentage of SCB length.

On total, six design variables are considered for double-SCB.

The centre of SCB ( 50 per cent of SCB length) will be positioned where the flow speed transits from supersonic to subsonic.

\subsubsection{Implementation}

The following conditions are for MSES coupled to the multi-resolution/population hierarchical populations.
1. First layer: Population size of ten with a computational grid of $36 \times 213$ points (Node 0 ).

2. Second layer: Population size of 20 with a computational grid of $24 \times 131$ points (Node1, Node2).

3. Third layer: Population size of 20 with a computational grid of $36 \times 111$ points (Node3Node6).

Note: these grid conditions produce less than 5 per cent accuracy error compared to precise model at the first layer (Node0).

\subsubsection{Numerical results}

As illustrated in Fig. 9, the algorithm was allowed to run for $24 \mathrm{~h}$ and 2508 function evaluations using a single $4 \times 2.8 \mathrm{GHz}$ processor and convergence occurred at 1053 function evaluations with $C d_{\text {Total }}=0.03441$ after $10 \mathrm{~h}$. 


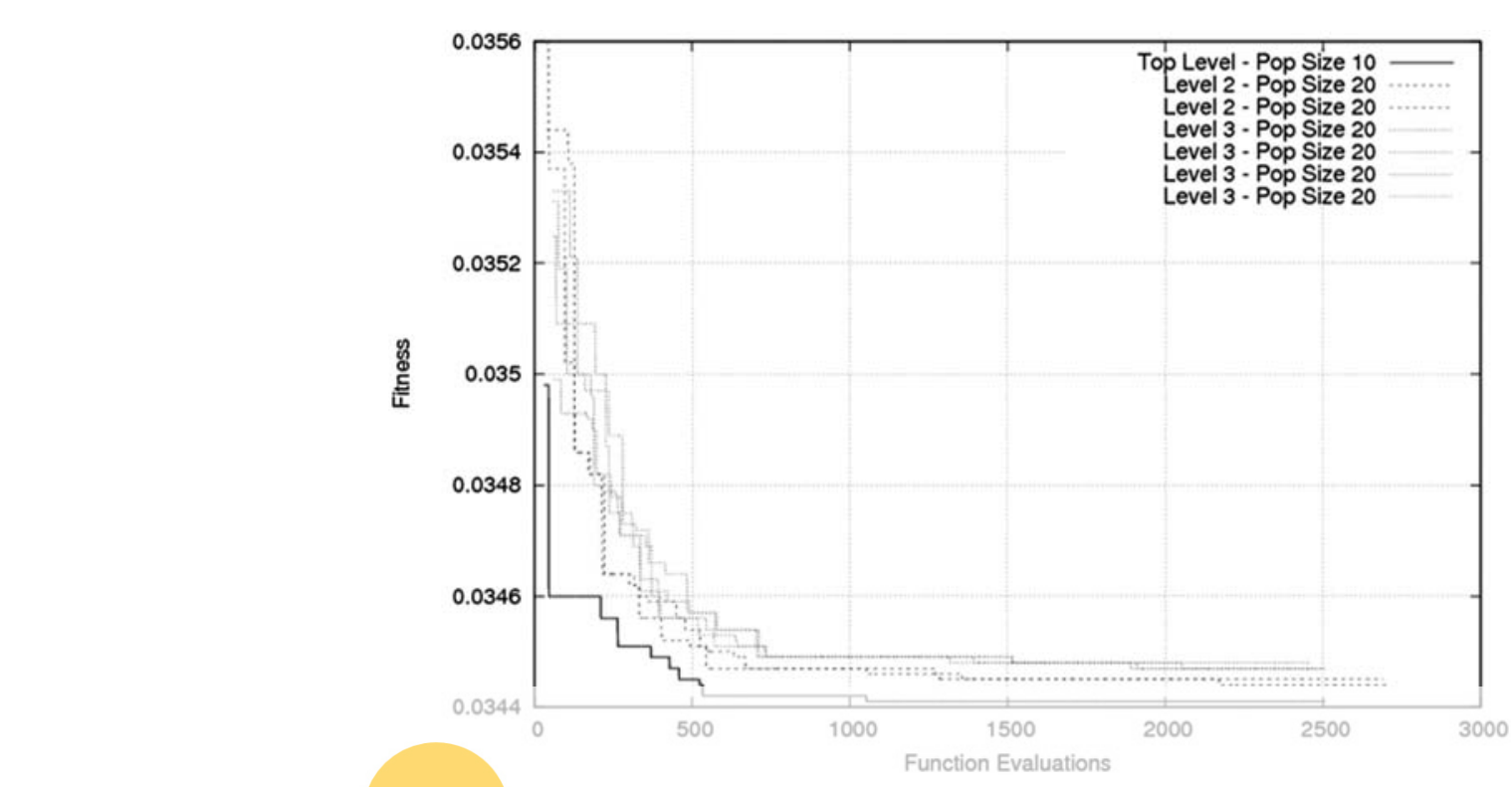

Fig. 9 Convergence history obtained by HAPMOEA
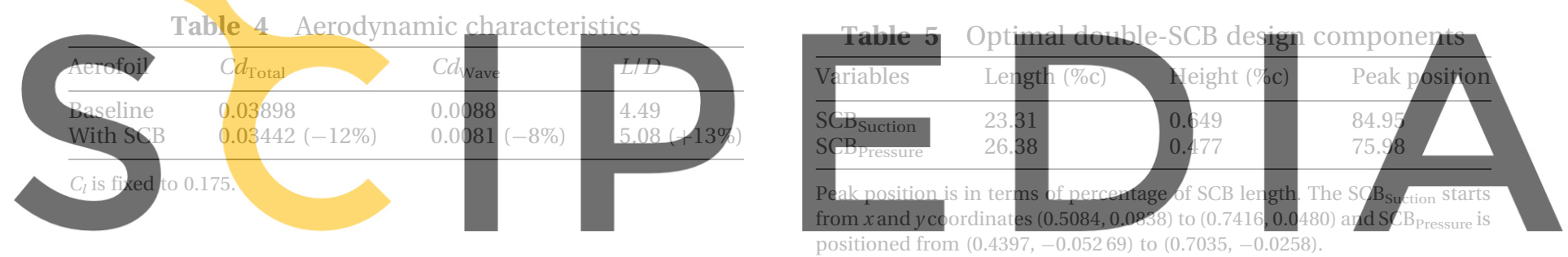

Register for free at https//www.scipedia.com to download the version without the watermark

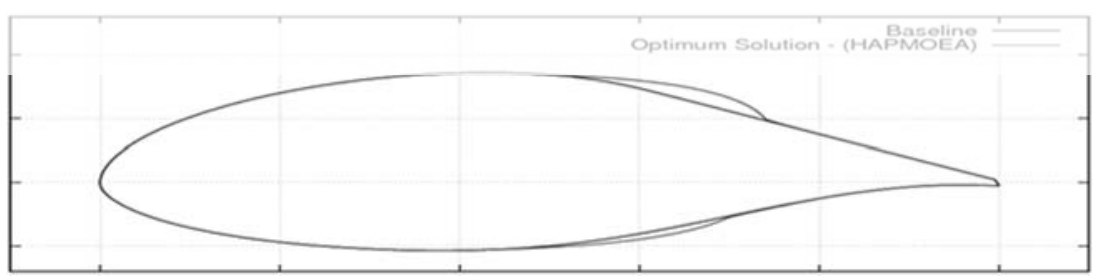

Fig. 10 Baseline design with the optimal double-SCB obtained by HAPMOEA (Note: $\max t / c=0.14$ at $41 \% \mathrm{c}$ and $\max$ camber $=0.0209$ at $69.8 \% \mathrm{c}$ )

Table 4 compares the aerodynamic characteristics obtained by the baseline design (RAE 5243) and the baseline design with SCBs on both the suction and pressure sides. Applying SCB to RAE 5243 aerofoil saves the wave drag by 8 per cent which leads 12 per cent of total drag reduction. This optimal double-SCB improves $L / D$ by 13.0 per cent.

The optimal shape of double-SCB is described in Table 5. Figure 10 compares the geometry of the baseline design and baseline with the optimal double-SCB which has same $t / c$ while the max camber (max, maximum) is increased by 0.0005 and its position is moved $16 \% \mathrm{c}$ towards to the trailing edge when compared to the baseline design.

Figure 11 shows the contour of baseline design with the optimal double-SCB. It can be seen that the strong shocks on the baseline design shown in Fig. 7(b) get weaker by adding double-SCB.

Figure 12 compares the $C p$ distribution obtained by the baseline design and the baseline design with SCBs on the suction and pressure sides. 


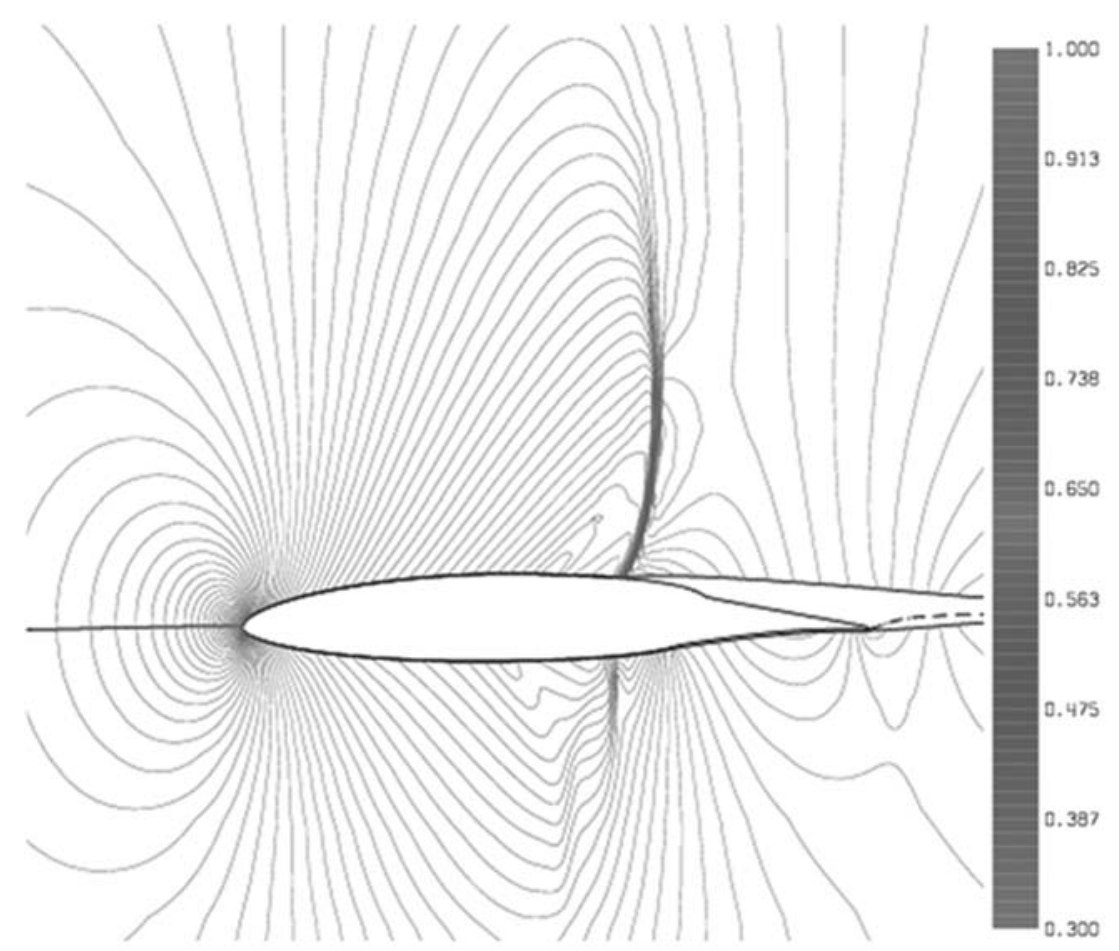

Fig. $11 P / P_{0}$ contour of the optimal double-SCB solution obtained by HAPMOEA

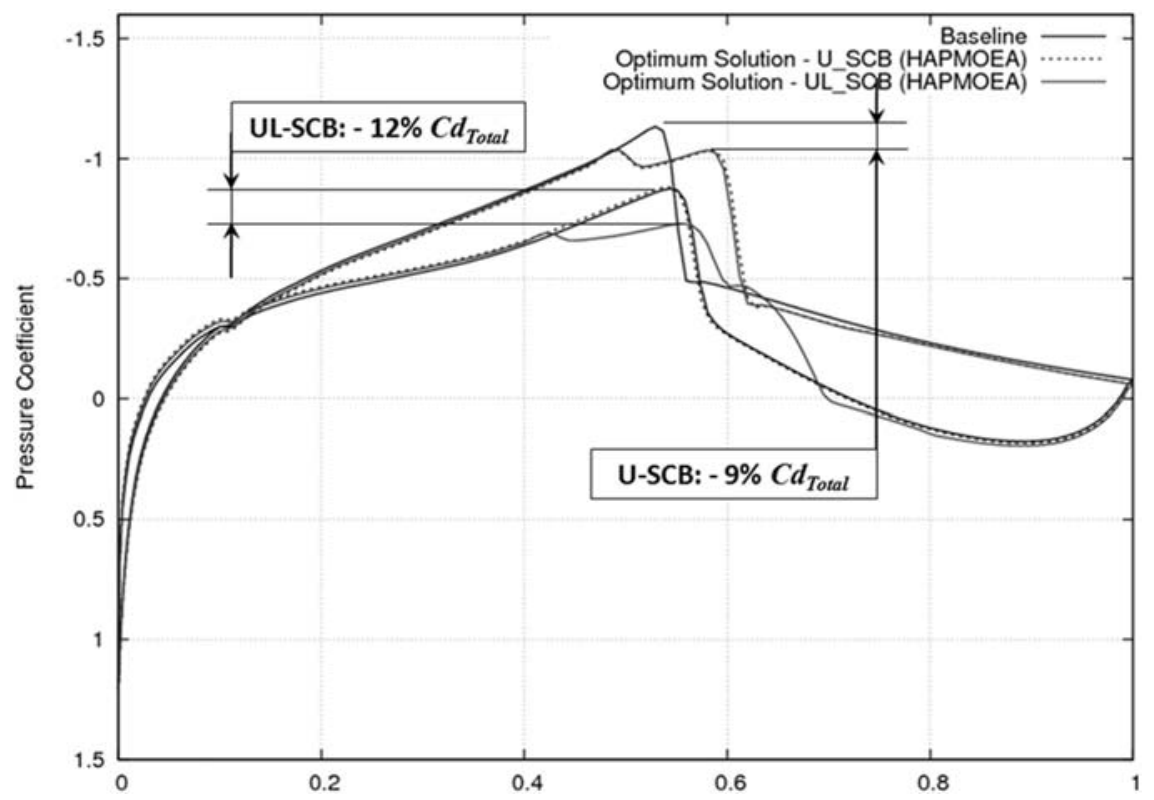

Fig. $12 C p$ distributions obtained by the baseline design and the optimal solution (U-SCB and UL-SCB); U-SCB and UL-SCB represent the optimal SCB on the suction side only and the optimal SCBs on both the suction and pressure sides of aerofoil respectively

It can be seen that the total drag is reduced by 9 per cent while the double SCB reduces 12 per cent of total drag. The shock on the suction side is delayed while the shock on the pressure side becomes weak isentropic waves.

\subsection{SCB design optimization using Hybrid-Game 5.3.1 Problem definition}

This test case considers a single-objective doubleSCB design optimization using Hybrid-Game on 
Table 6 Design variable distribution for Hybrid-Game

\begin{tabular}{lcccc}
\hline & & Hybrid-Game & NP2 & $\begin{array}{l}\text { Hierarchical asynchronous parallel multi-objective } \\
\text { evolutionary algorithm (Nodes 0-6) }\end{array}$ \\
\cline { 2 - 4 } Type of SCB & GP & NP1 & $\checkmark$ & $\checkmark$ \\
\hline SCB Suction & $\checkmark$ & $\checkmark$ & $\checkmark$ & $\checkmark$ \\
\hline
\end{tabular}

GP, NP1, and NP2 represent global player and Nash-Players 1 and 2.

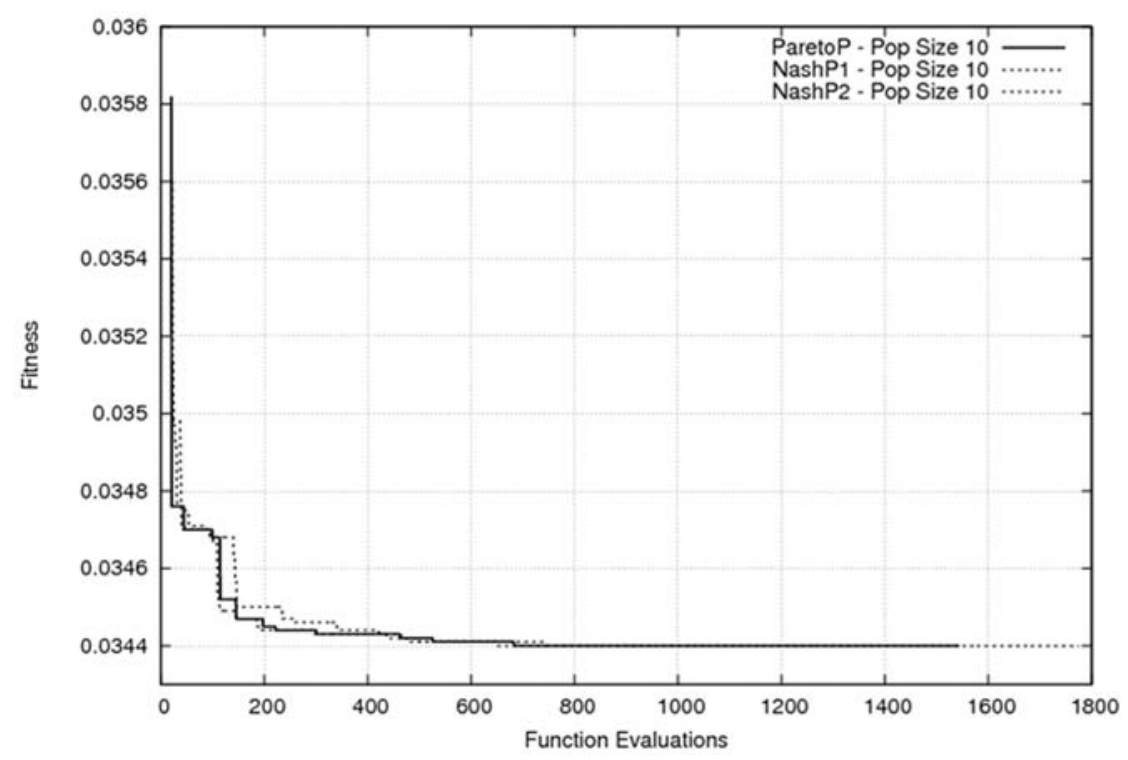

Fig. 13 Convergence history obtained by Hybrid-Game

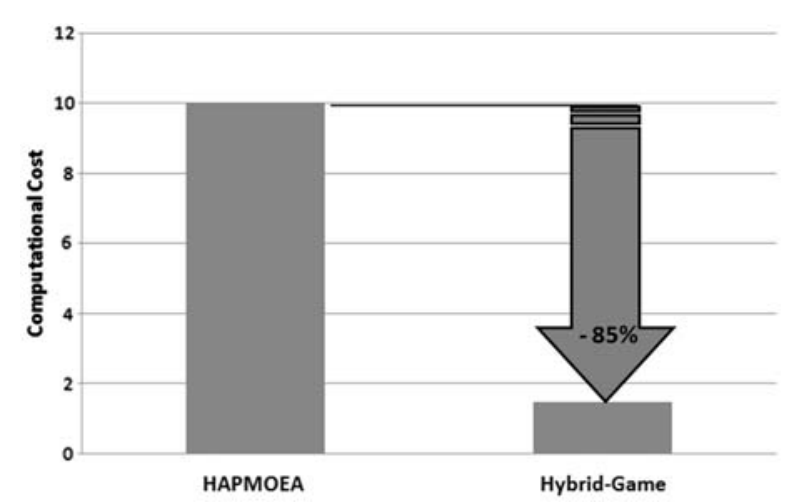

Fig. 14 Performance comparison between HAPMOEA and Hybrid-Game

Table 7 Aerodynamic characteristics

\begin{tabular}{llll}
\hline Aerofoil & $C d_{\text {Total }}$ & $C d_{\text {Wave }}$ & $L / D$ \\
\hline Baseline & 0.03898 & 0.0088 & 4.49 \\
With SCB & $0.03437(-12 \%)$ & $0.0081(-8 \%)$ & $5.09(+13 \%)$ \\
\hline
\end{tabular}

$C_{l}$ is fixed to 0.175 .
Table 8 Optimal SCB design variables obtained by Hybrid-Game

\begin{tabular}{|c|c|c|c|}
\hline Variables & Length $(\% \mathrm{c})$ & Height (\%c) & Peak position \\
\hline $\mathrm{SCB}_{\text {Suction }}$ & 23.65 & 0.649 & 84.99 \\
\hline $\mathrm{SCB}_{\text {Pressure }}$ & 23.88 & 0.384 & 80.35 \\
\hline
\end{tabular}

Peak position is in terms of percentage of SCB length. SCB Suction $_{\text {and }}$ $\mathrm{SCB}_{\text {Pressure }}$ represent SCB on the suction and pressure sides of RAE 5243 aerofoil. The $\mathrm{SCB}_{\text {Suction }}$ starts from $x$ and $y$ coordinates $(0.5067,0.0839)$ to

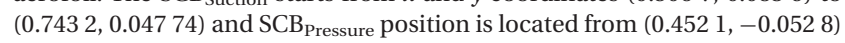
to $(0.6910,-0.0277)$.

MOEA to minimize total drag at flow conditions $M_{\infty}=0.8, C_{l}=0.175$, and $R e=18.63 \times 10^{6}$. HybridGame consists of three players: one Global-Player (GP), two Nash-Players (NP1 and NP2) instead of hierarchical multi-population/resolution (Node0Node6). The fitness functions for Hybrid-Game are shown in equation (8).

$$
\begin{aligned}
& f_{\mathrm{GP}}\left(\mathrm{SCB}_{\text {Suction }}, \mathrm{SCB} \mathrm{B}_{\text {Pressure }}\right)=\min \left(C d_{\text {Total }}\right) \\
& f_{\mathrm{NP} 1}\left(\mathrm{SCB}_{\text {Suction }}, \mathrm{SCB}_{\text {Pressure }}^{*}\right)=\min \left(C d_{\text {Total }}\right) \\
& \left.f_{\mathrm{NP} 2} S C B_{\text {Suction }}^{*}, \mathrm{SCB}_{\text {Pressure }}\right)=\min \left(C d_{\text {Total }}\right)
\end{aligned}
$$




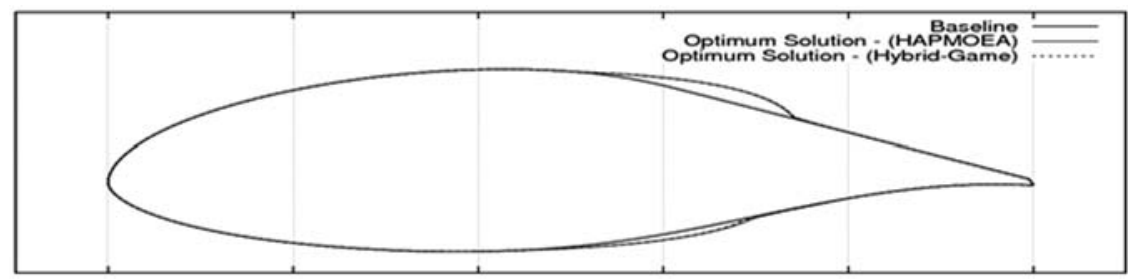

Fig. 15 Baseline design with the optimal double-SCB obtained by Hybrid-Game (Note: max $t / c=0.14$ at $41 \% \mathrm{c}$ and max camber $=0.0214$ at $69.0 \% \mathrm{c}$ )

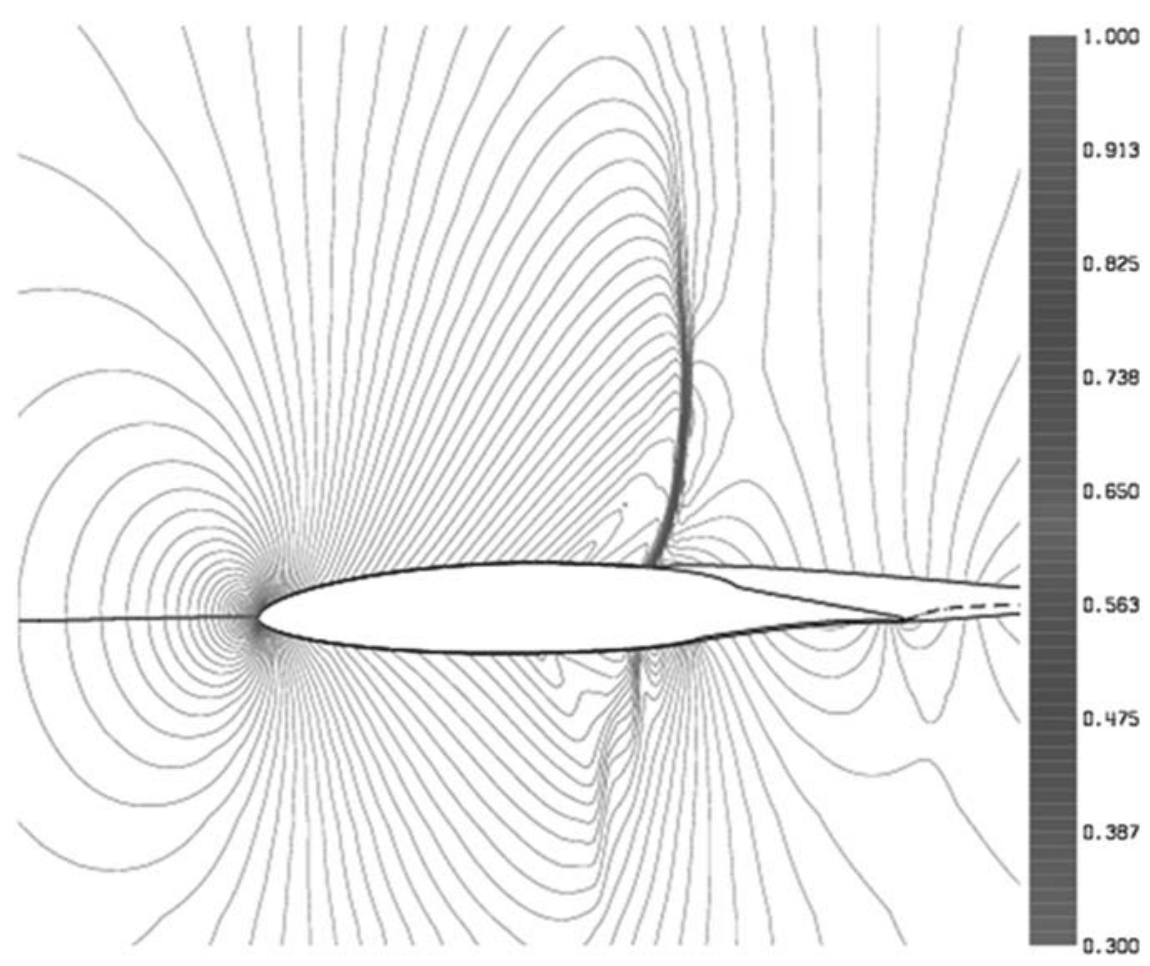

Fig. $16 P / P_{0}$ contour of the optimal double-SCB solution obtained by Hybrid-Game

where $C d_{\text {Total }}=C d_{\text {Viscous }}+C d_{\text {Wave }}$. $\mathrm{SCB}_{\text {Suction }}$ and $\mathrm{SCB}_{\text {Pressure }}$ represent $\mathrm{SCB}$ on the suction side and pressure side, and * the elite SCB design obtained by Nash-Players. SCB Suction and $\mathrm{SCB}_{\text {Pressure }}^{*}$ are the elite SCB designs obtained by Nash-Players 1 and 2. These elite SCB designs will be seeded to the population of Global-Player at every ten function evaluations and will act as a pre-conditioner.

\subsubsection{Design variables}

The design variable bounds for the upper and lower SCB geometries are illustrated in Table 3. Table 6 shows design variable distribution for Hybrid-Game. It can be seen that the Nash-Players 1 and 2 consider only three design variables while the Global-Player of Hybrid-Game considers six design variables.

\subsubsection{Implementation}

The following conditions are for MSES coupled to Hybrid-Game: Global-Player, Nash-Player 1, and Nash-Player 2:

(a) GP: Population size of ten with a grid of $36 \times 213$;

(b) NP1: Population size of ten with a grid of $36 \times 213$;

(c) NP2: Population size of ten with a grid of $36 \times 213$.

\subsubsection{Numerical results}

As illustrated in Fig. 13, the algorithm was allowed to run for $5 \mathrm{~h}$ and 1775 function evaluations using single $4 \times 2.8 \mathrm{GHz}$ processor and convergence occurred at 683 function evaluations (approximately $1.9 \mathrm{~h}$ ) with $C d_{\text {Total }}=0.0344$ which HAPMOEA could not capture 

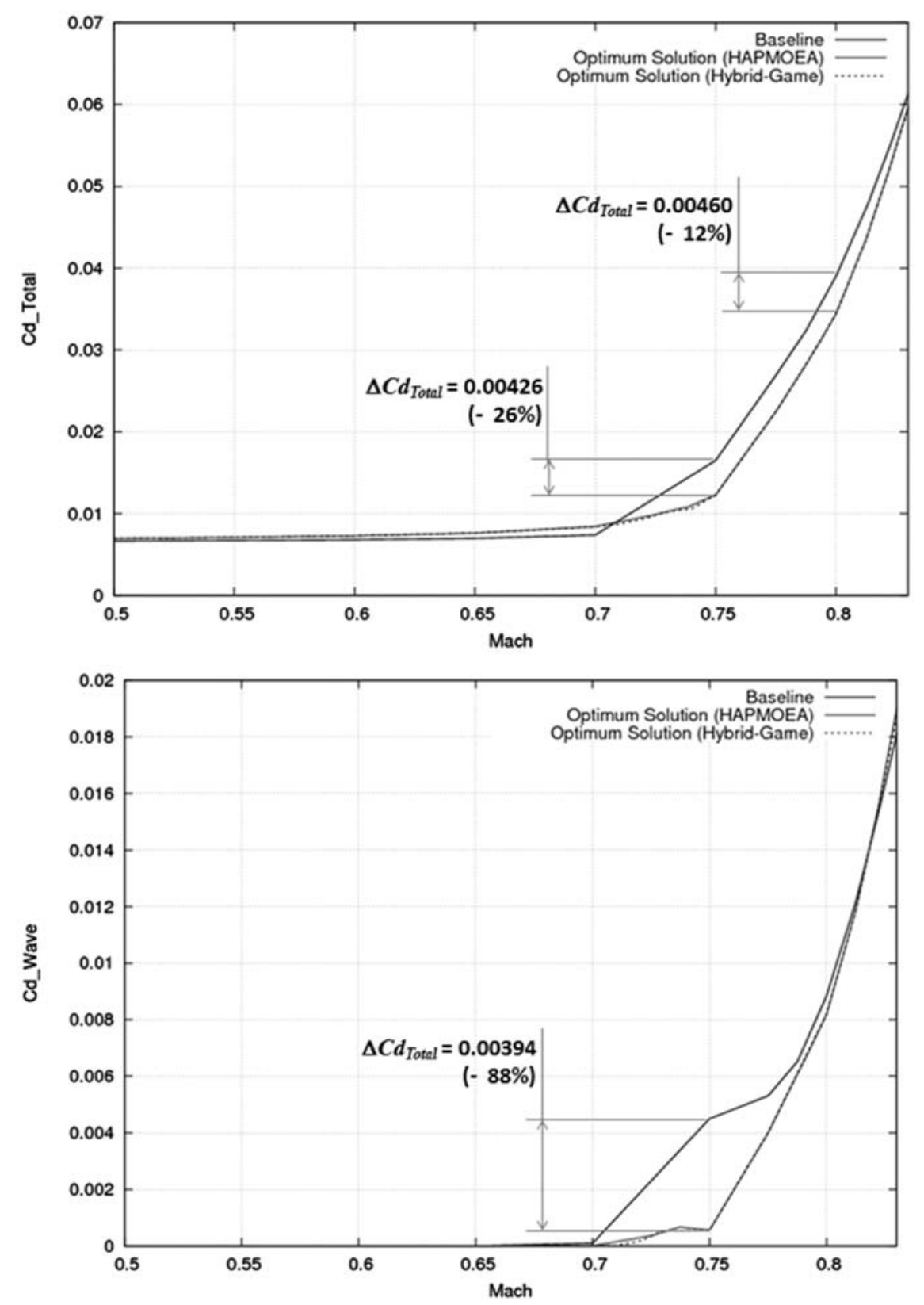

Fig. 17 (a) $C d_{\text {Total }}$ versus Mach numbers and $C d_{\text {Wave }}$ versus Mach numbers

even after $24 \mathrm{~h}$ shown in previous test Section B. To compare the computational efficiency of HAPMOEA and Hybrid-Game, the fitness value is chosen to be $C d_{\text {Total }}=0.03441$ which HAPMOEA captured after $10 \mathrm{~h}$. Hybrid-Game took $1.48 \mathrm{~h}$ which is only 15 per cent of HAPMOEA computational cost. In other words, Nash-Game improves the performance of EA by 85 per cent, as shown in Fig. 14.

Table 7 compares the aerodynamic characteristics obtained by the baseline design (RAE 5243) and the baseline design with SCBs on the suction and pressure sides. Applying SCB to RAE 5243 aerofoil saves the wave drag by 8 per cent which leads to 12 per cent of total drag reduction. This optimal double-SCB improves $L / D$ by 13.0 per cent.

The optimal double shape of double-SCB obtained by Hybrid-Game is described in Table 8. It can be seen that the $\mathrm{SCB}_{\text {Suction }}$ obtained by Hybrid-Game and HAPMOEA (Table 5) have almost same shape while the $\mathrm{SCB}_{\text {Pressure }}$ from Hybrid-Game is 10 per cent shorter than the one obtained by HAPMOEA. 

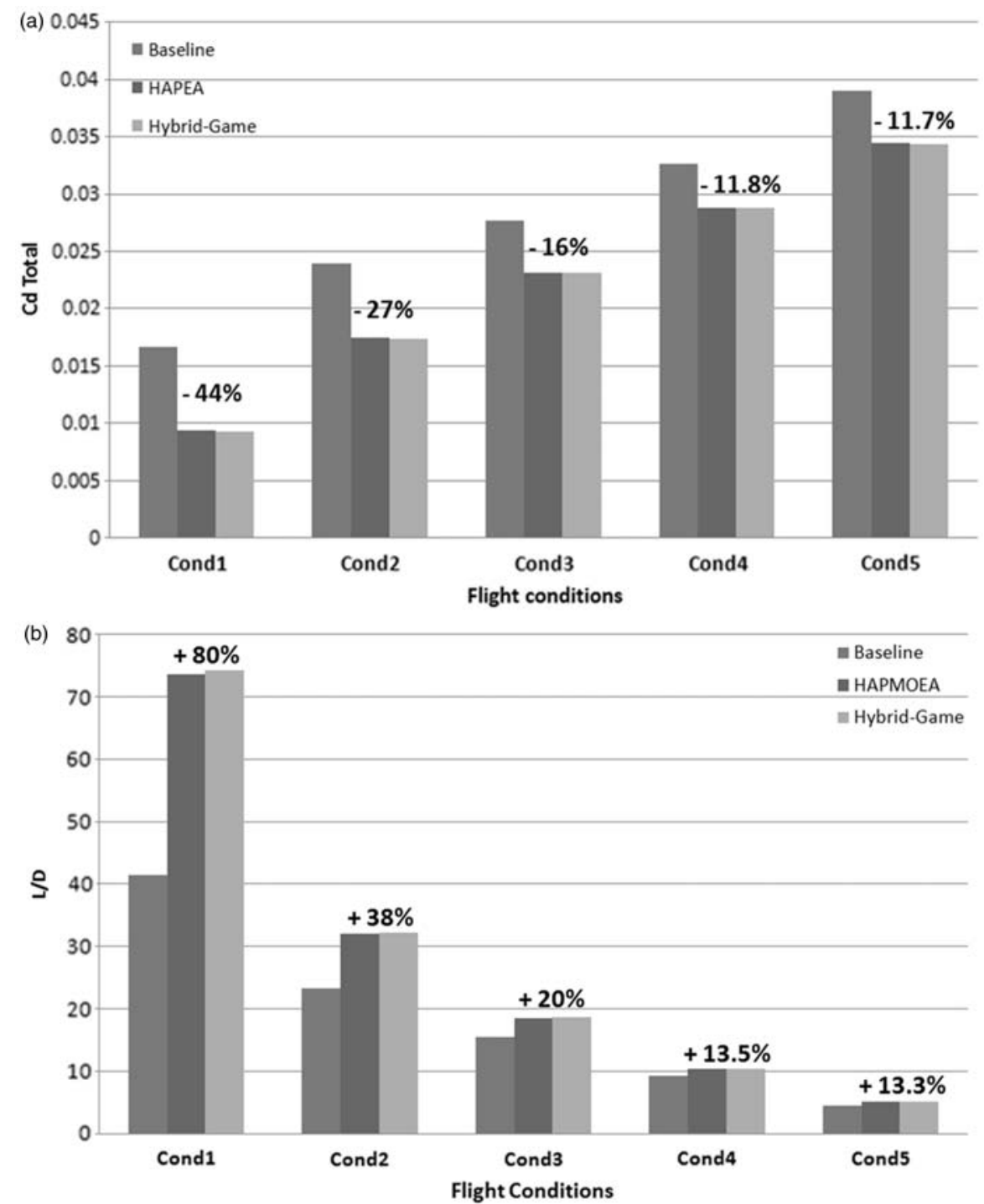

Fig. 18 (a) Drag reduction obtained by the optimal double-SCB at five different flight conditions

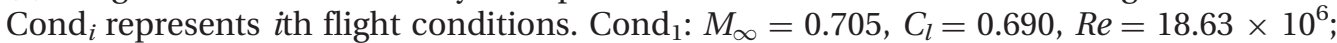
Cond $_{2}: M_{\infty}=0.730, C_{l}=0.560, R e=18.63 \times 10^{6} ;$ Cond $_{3}: M_{\infty}=0.750, C_{l}=0.430$, $R e=18.63 \times 10^{6} ;$ Cond $_{4}: M_{\infty}=0.775, C_{l}=0.300, R e=18.63 \times 10^{6} ;$ and Cond $_{5}:$ $M_{\infty}=0.800, C_{l}=0.175, R e=18.63 \times 10^{6}$ (b) $L / D$ obtained by the optimal double-SCB at five different flight conditions

Figure 15 illustrates the geometry of the baseline design and baseline with the optimal double-SCB from HAPMOEA and Hybrid-Game. The baseline design with double-SCB obtained by Hybrid-Game has same $t / c$ while the max camber is increased by 0.00055 and its position is moved $15 \%$ c towards to the trailing edge when compared to the baseline design.

Figure 16 shows the pressure contour of baseline design with the optimal double-SCB obtained by Hybrid-Game. It can be seen that the upper shock is moved towards to the trailing edge while lower shock becomes weak isentropic waves.
Figure 17(a) and (b) compares total drag ( $\left.C d_{\text {Total }}\right)$ and wave drag ( $\left.C d_{\text {Wave }}\right)$ distributions obtained by the baseline design and with the optimal double SCB from both HAPMOEA and Hybrid-Game. The flow conditions are $M_{\infty} \in$ [0.5:0.85] with constant $C l_{\text {Fixed }}=0.175$ and $R e=18.63 \times 10^{6}$. It can be seen that both optimal double-SCBs obtained by HAPMOEA and Hybrid-Game perform almost same drag along the Mach numbers. The baseline design with the optimal double-SCB starts to produce lower total drag when Mach number is higher than 0.71. One thing should be noticed from Fig. 17(b) is that the critical Mach number $\left(M_{\mathrm{C}}=0.65\right)$ for baseline 

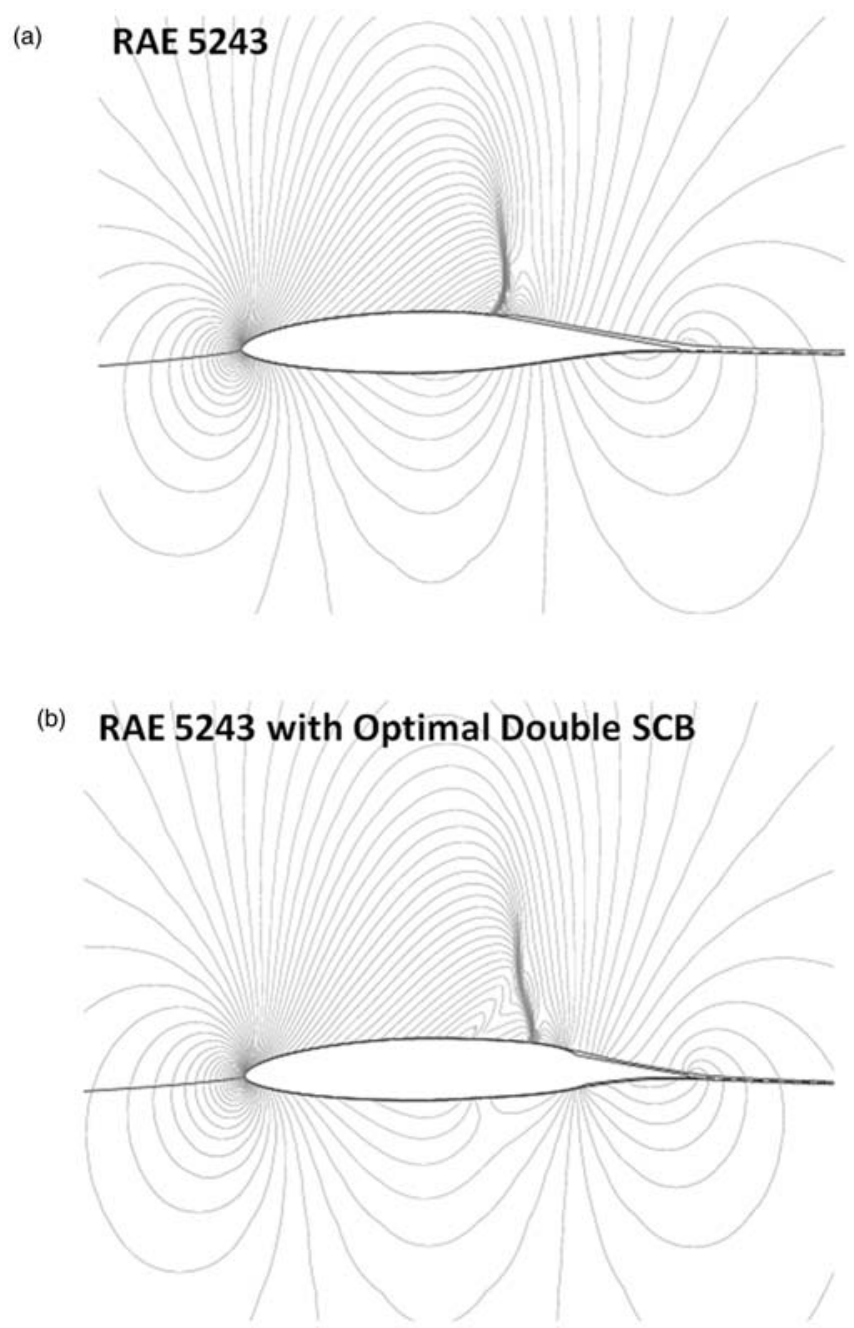

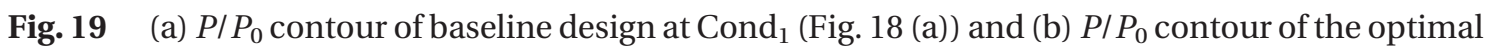
double-SCB obtained by Hybrid-Game at Cond ${ }_{1}$ (Fig. 18(a))

design is extended to 0.71 by adding double SCB. The maximum total drag reduction ( -26 per cent) is observed at $M_{\infty}=0.75$, as shown in Fig. 17(a), due to 88 per cent of wave drag reduction shown in Fig. 17 (b) when compared to the baseline design.

The optimal double-SCB obtained by HAPMOEA and Hybrid-Game is also tested at five different flight conditions. The histogram showed in Fig. 18(a) compares the total drag. It can be seen that the double-SCB optimized at critical flight conditions reduces more total drag by 15 per cent to 44 per cent while improving the lift-to-drag ratio by 13.5 per cent to 80 per cent, as shown in Fig. 18(b) at the normal flight conditions.

One example $\left(\right.$ Cond $\left._{1}\right)$ is shown in Fig. 19(a) and (b) where the pressure ratio contours obtained by the baseline design and with the optimal double-SCB solution from the Hybrid-Game are illustrated. Even though the double-SCB is optimized at the critical flight condition, the optimal double-SCB moves the normal strong shock, as shown in Fig. 19(a) towards to the trailing edge by $10 \% \mathrm{c}$ and reduce the total drag by 44 per cent which leads to 80 per cent improvement of $L / D$.

To summarize the optimization test case, doubleSCB on RAE 5243 is optimized using HAPMOEA and Hybrid-Game to reduce transonic drag at the critical flight conditions. The use of optimal double SCB is beneficial at both normal and critical flow conditions. In addition, Hybrid-Game significantly reduces the computational cost for double-SCB design optimization while generating high-quality optimal solution when compared to HAPMOEA.

The design engineer will choose the optimal doubleSCB obtained by Hybrid-Game which has 10 per cent shorter length than SCB from HAPMOEA. In other 
words, the double-SCB from Hybrid-Game will require less modification in current manufacturing system as well as less material.

\section{CONCLUSION}

In this article, two advanced optimization techniques have been demonstrated and implemented as a methodology for AFC bump named as SCB shape design optimization. Analytical research clearly shows the benefits of using Hybrid-Game in terms of computational cost and design quality. In addition, the use of SCB on current aerofoil reduces significantly the transonic drag. In long-term view, the use of SCB will not only save operating cost but also critical aircraft emissions due to less fuel burn.

Future work will focus on robust multi-objective design optimization of SCB (Taguchi method) which can produce the model with better performance and stability at variability of operating conditions and transition positions. In forthcoming research, other evolutionary optimizers including strength Pareto evolutionary algorithm 2, self-adaptive Pareto differential evolution will be hybridized with Nash-Game strategy and their results will be compared in terms of solution quality and computational cost.

\section{FUNDING}

This study has been supported by the Spanish Ministerio de Ciencia e Innovación through project DPI2008-05250.

\section{ACKNOWLEDGEMENTS}

The authors gratefully acknowledge E. J. Whitney and M. Sefrioui, Dassault Aviation for their fruitful discussions on Hierarchical EAs and their contribution to the optimization procedure and, also to M. Drela at MIT for providing MSES software. The authors thank Ning Qin at University of Sheffield for his fruitful discussion on SCB.

\section{(C) Authors 2011}

\section{REFERENCES}

1 Lee, D. S., Periaux, J., and Gonzalez, L. F. UAS mission path planning system (mpps) using hybrid-game coupled to multi-objective optimiser. J. Dyn. Syst. Meas. Contr., 2010, 132(4), 1-11. (041005).

2 Deb, K., Agrawal, S., Pratap, A., and Meyarivan, T. A fast and elitist multi-objective genetic algorithm: NSGA-II. IEEE Trans. Evol. Comput., 2002, 6(2), 182-197.
3 Lee, D. S., Gonzalez, L. F., Periaux, J., and Srinivas, K. Hybrid-game strategies coupled to evolutionary algorithms for robust multidisciplinary design optimization in aerospace engineering. IEEE Trans. Evol. Comput., 2011, 15(2), 133-150 (TEVC00213-2009) (In Press).

4 Periaux, J., Lee, D. S., Gonzalez, L. F., and Srinivas, K. Fast reconstruction of aerodynamic shapes using evolutionary algorithms and virtual nash strategies in a CFD design environment. Special Issue J. Comput. Appl. Math. (JCAM), 2009, 232(1), 61-71. ISSN 0377-0427.

5 Lee, D. S., Gonzalez, L. F., and Whitney, E. J. Multiobjective, Multidisciplinary Multi-fidelity Design tool: HAPMOEA - User Guide Appendix-I, D.S. Lee. Uncertainty Based Multiobjective and Multidisciplinary Design Optimization in Aerospace Engineering, 2007 (The Univ. of Sydney, Sydney, NSW, Australia).

6 Bart-Smith, H. and Risseeuw, P. E. High authority morphing structures. In Proceedings of IMECE 03, no. IMECE 2003-43377, ASME International Mechanical Engineering Congress, 2003 (Washington, DC) November.

7 Oborn, R., Kota, S., and Hetrick, J. A. Active flow control using high-frequency compliant structures. J. Aircr., 2004, 41 (3), 603-609.

8 Ashill, P. R., Fulker, L. J., and Shires, A. A novel technique for controlling shock strength of laminar-flow aerofoil sections. In Proceedings of 1st Europian Forum on Laminar flow technology, 1992, pp. 175-183 (DGLR, AAAF, RAeS, Hamburg, Germany), 16-18 March.

9 Qin, N., Zhu, Y., and Shaw, S. T. Numerical study of active shock control for transonic aerodynamics. Int. J. Numer. Methods Heat Fluid Flow, 2004, 14(4), 444-466.

10 Fulker, J. L. and Simmons, M. J. An experiment study of shock control methods, 1994 (Technical Report, DERA), DRA/AS/HWA/TR94007/1.

11 An Open Database Workshop for Multiphysics Software Validation, TA5: Shock control bump optimisation on a transonic laminar flow airfoil (Chairman N. Qin). Univ. Jyvaskyla, Finland, 18 December 2009 \& 10-12 March 2010.

12 Hansen, N. and Ostermeier, A. Completely derandomized self-adaptation in evolution strategies. Evol. Comput., 2001, 9(2), 159-195.

13 Hansen, N., Möller, S. D., and Koumoutsakos, P. Reducing the time complexity of the derandomized evolution strategy with covariance matrix adaptation (CMA-ES). Evol. Comput., 2003, 11(1), $1-18$.

14 Wakunda, J. and Zell, A. Median-selection for parallel steady-state evolution strategies. In parallel problem solving from nature, PPSN VI (Eds M. Schoenauer, K. Deb, G. Rudolph, X. Yao, E. Lutton, J. J. Merelo, H.-P. Schwefel), 2000, pp. 405-414 (Springer, Berlin).

15 Van Veldhuizen, D. A., Zydallis, J. B., and Lamont, G. B. Considerations in engineering parallel 
multiobjective evolutionary algorithms. IEEE Trans. Evol. Comput., 2003, 7(2), 144-217.

16 Sefrioui, M. and Périaux, J. A hierarchical genetic algorithm using multiple models for optimization. In Parallel problem solving from nature, PPSN VI (Eds M. Schoenauer, K. Deb, G. Rudolph, X. Yao, E. Lutton, J. J. Merelo, H.-P. Schwefel), 2000, pp. 879-888 (Springer, Berlin).

17 Lee, D. S. Uncertainty based multiobjective and multidisciplinary design optimization in aerospace engineering, 2008, pp. 348-370 (The University of Sydney, Sydney, NSW, Australia), Section 10.7.

18 Zitzler, E., Deb, K., and Thiele, L. Comparison of multiobjective evolutionary algorithms: Empirical results. Evol. Comput., 2000, 8(2), 173-195.

19 Drela, M. A user's guide to MSES 2.95, 1996 (MIT Computational Aerospace Sciences Laboratory) September. 